EPJ Web of Conferences 64, 06001 (2014)

DOI: $10.1051 /$ epjconf/ 20146406001

(C) Owned by the authors, published by EDP Sciences, 2014

\title{
The Magnetospheres of (Accreting) Neutron Stars
}

\section{Observational Clues}

J. Wilms ${ }^{1, a}$

${ }^{1}$ Remeis Sternwarte \& Erlangen Centre for Astroparticle Physics, Sternwartstr. 7, 96049 Bamberg, Germany

\begin{abstract}
I give an overview of the most important observational tools to study the magnetospheres of accreting neutron stars, with a focus on accreting neutron stars in high mass X-ray binary systems. Topics covered are the different types of accretion onto neutron stars and the structure of the accretion column, and how models for these can be tested with observations.
\end{abstract}

\section{Introduction}

Accreting neutron stars in X-ray binaries provide direct observational information on the interaction of their strong $\left(10^{12} \mathrm{G}\right)$ magnetic fields with the stellar wind of their donor stars. Through observations of cyclotron lines - absorption line like features caused by the interaction of hard X-ray photons with quantized electrons in the magnetic field at the neutron star's magnetic poles - these objects are also our only opportunity for direct measurements of the strength of neutron star magnetic fields.

The aim of this review is to give an overview of the main properties and physical ideas of these objects for astrophysicists who do not specialize on neutron stars and to serve as an introduction to the more specialized contributions in these proceedings. In Sect. 2 I discuss the different ways how neutron stars in X-ray binary systems accrete matter from their donor stars, while Sect. 3 is devoted to a description of the luminosity-dependent modes of the accretion column and how these can be tested using observations of cyclotron lines. For space reasons I will only briefly touch on subjects that are discussed elsewhere in this volume, such as ideas that the accretion column in neutron stars is several kilometers in length (leading to $B$-fields of $\sim 10^{14} \mathrm{G}$ at the magnetic poles; see the contribution by Doroshenko et al.) and models explaining the cyclotron line variability as reflection from the neutron star surface (see the contribution by Mushtukov et al.).

\section{Accretion in High Mass X-ray Binaries}

\subsection{Theoretical Overview}

Accreting neutron stars are found in three different kinds of X-ray binaries. In low-mass X-ray binaries (LMXB) the donor star is a late type star. Accretion is mainly via Roche lobe accretion and an extended accretion disk [1]. LMXB are typically old systems and therefore the magnetic fields of the

\footnotetext{
a e-mail: joern.wilms@sternwarte.uni-erlangen.de
} 


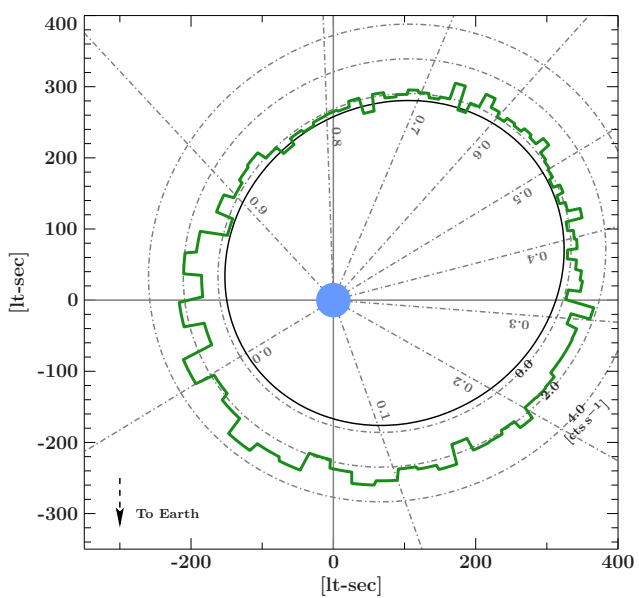

Figure 1. Orbit of the Be X-ray binary EXO 2030+375 according to the orbital parameters of Wilson et al. [3] and assuming $10 R_{\odot}$ for the $\mathrm{B} 0$ Ve donor star, a typical value for B0 stars [4]. The green line shows the average phase dependent RXTE-ASM 2-10 keV count rate during for the time interval MJD 54392-53860, i.e., during the normal outbursts before its 2006 giant outburst. Note that the $\mathrm{X}$-ray count rate increases slightly before the periastron, where accretion starts, and that the accretion continues until close to apastron.

neutron stars are expected to have decayed. Extensive magnetospheres are therefore rare, although there are examples of neutron stars with $10^{12} \mathrm{G}$ fields which are embedded in an accretion disk such as Her X-1 [e.g., 2].

Most neutron stars with strong magnetic fields are therefore located in high mass X-ray binaries (HMXB). Here the donor star has spectral type O or B. In HMXB the accreted matter originates from the stellar wind of the donor star [5-7, and references therein]. Most of these systems have rather tight orbits with orbital periods of the order of a week. Their luminosity is to first order set by the mass accretion through the Bondi radius [see, e.g., 8],

$$
r_{\mathrm{B}} \sim \frac{2 G M}{v^{2}}=3.8 \times 10^{10} \mathrm{~cm} \frac{M}{1.44 M_{\odot}}\left(\frac{v}{1000 \mathrm{~km} \mathrm{~s}^{-1}}\right)^{2}
$$

where $M$ is the mass of the neutron star, and $v$ the relative speed between the neutron star and the stellar wind [9]. The fraction of the stellar wind accreted is then $[10,11]$

$$
\frac{\dot{M}}{\dot{M}_{\text {star }}} \sim \frac{r_{\mathrm{B}}^{2}}{4 a} \sim 2 \times 10^{-5} \frac{v}{1000 \mathrm{~km} \mathrm{~s}^{-1}}\left(\frac{P_{\text {orb }}}{10 \mathrm{~d}}\right)^{-4 / 3}\left(\frac{M}{30 M_{\odot}}\right)^{-2 / 3}
$$

where $a$ is the orbital separation of the neutron star and its donor. Note that mass loss rates from early type stars can reach up to $10^{-6} M_{\odot}$ year ${ }^{-1}$, i.e., even if the neutron star accretes only a small fraction of the total stellar wind, it can radiate close to its Eddington limit ${ }^{1}$.

A sub type of HMXB are the Be binaries [12-14], where the neutron star is in an eccentric orbit, with typical orbital periods of weeks to months. Accretion from the Be disk can be triggered during periastron passages of the neutron star (Fig. 1). Depending on the Be star's mass loss rate, outbursts can be recurrent during each periastron passage [e.g., in GRO J1008-57 15]. Other sources show outbursts sporadically on timescales of years or decades. For example, the Be binary XTE J1946+274 showed a sequence of outbursts during each periastron passage in 1998-2001 [16], then went into a phase of quiescence, and reappeared with another sequence of outbursts in 2010/2011 [17].

A recently recognized third class of accreting, magnetized neutron stars are the symbiotic X-ray binaries, where the donor star is a M-giant. Only seven symbiotic X-ray binaries are known, of which GX $1+4$ is the most prominent [18-20]. With orbital periods of several $100 \mathrm{~d}$, the orbits of these

\footnotetext{
${ }^{1}$ For a $1.4 M_{\odot}$ neutron star, the Eddington mass accretion rate is $3 \times 10^{-9} M_{\odot}$ year $^{-1} / \eta$ where $\eta$ is the efficiency of the accretion process.
} 


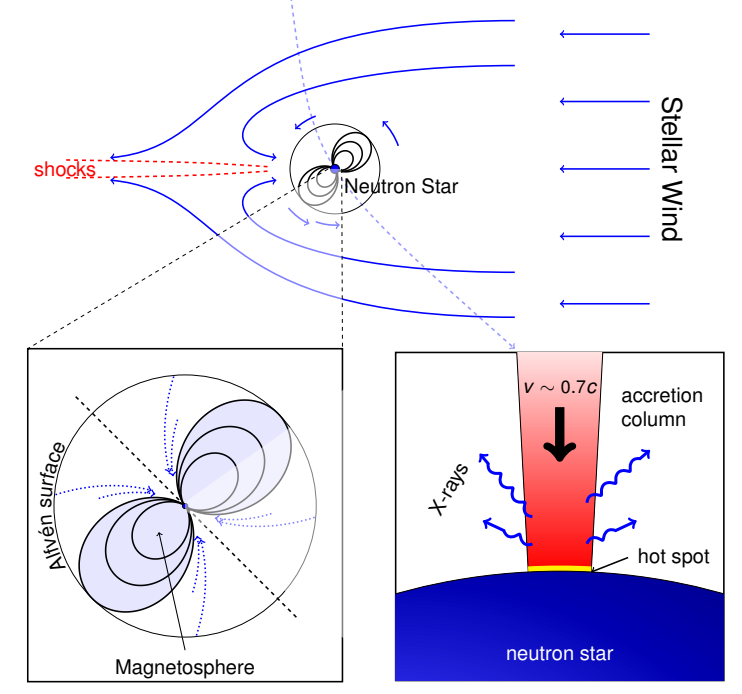

Figure 2. Wind accretion onto a neutron star [after 33]. After the stellar wind is captured by the neutron star's gravitational field, at a distance of $\sim 2000 \mathrm{~km}$ it couples to the neutron star's magnetic field and falls along the field lines onto the neutron star. Accretion columns form close to the surface of the neutron star where X-rays are produced. The rotation of the neutron star results in the appearance of the accreting neutron star as an X-ray pulsar.

systems are too wide to allow Roche Lobe overflow and therefore it is assumed that the neutron star is accreting from the wind of the M-star. With wind speeds of $\sim 100 \mathrm{~km} \mathrm{~s}^{-1}$ it is in principle possible to explain the typical luminosities of symbiotic X-ray binaries. While these speeds are significantly higher than those of typical M-star winds $\left(\sim 10 \mathrm{~km} \mathrm{~s}^{-1}\right)$, a speed of $250 \mathrm{~km} \mathrm{~s}^{-1}$ has been measured for the donor of GX $1+4$ [21]. With a rotation period of 5.5 hours(!), the symbiotic X-ray binary 3A $1954+319$ is the slowest rotating accreting X-ray pulsar [22, 23, and references therein]. The very long rotation periods and the observed period changes of symbiotics are difficult to reconcile with a standard wind accretion picture as they result in unphysical parameters for the systems such as $B$-field strengths above those of magnetars. Recently, Shakura et al. [24, see also [25]] have developed a new picture of magnetospheric accretion for these systems, which appears to deliver more reasonable neutron star parameters. See the contribution by these authors for more details.

\subsection{The Magnetosphere and the Accretion Flow}

Observations of accreting neutron stars allow us to study the physical surroundings of the neutron star as well as the interaction between the neutron star's magnetosphere and the accreting matter.

The structure of the stellar wind in which the neutron star is embedded can be measured by studying spectral features imprinted into the underlying non-thermal continuum of the neutron star's accretion column (see Sect. 3 below). The ionization state and structure of the accretion flow can be deduced from the time dependent variation of absorption features in the X-ray spectrum. In HMXB, the equivalent hydrogen column, $N_{\mathrm{H}}$, which is a measure of the amount of material along the line of sight, is variable on timescales down to minutes [e.g., 26, 27]. Strong fluorescent lines are evidence for the photoionization of the stellar wind by the strong X-ray source moving through it [e.g., 28-32].

Once the accreted material is in the sphere of influence of the neutron star it will shed its angular momentum and fall onto the compact object. Close to the neutron star, the gas motion will be dominated by the effects of the neutron star's strong magnetic field (Fig. 2). The radius where the $B$-field becomes important is roughly the Alfvén radius,

$$
r_{\mathrm{mag}}=\left(\frac{8 \pi^{2}}{G}\right)^{1 / 7}\left(\frac{R^{12} B^{4}}{M \dot{M}^{2}}\right)^{1 / 7}
$$




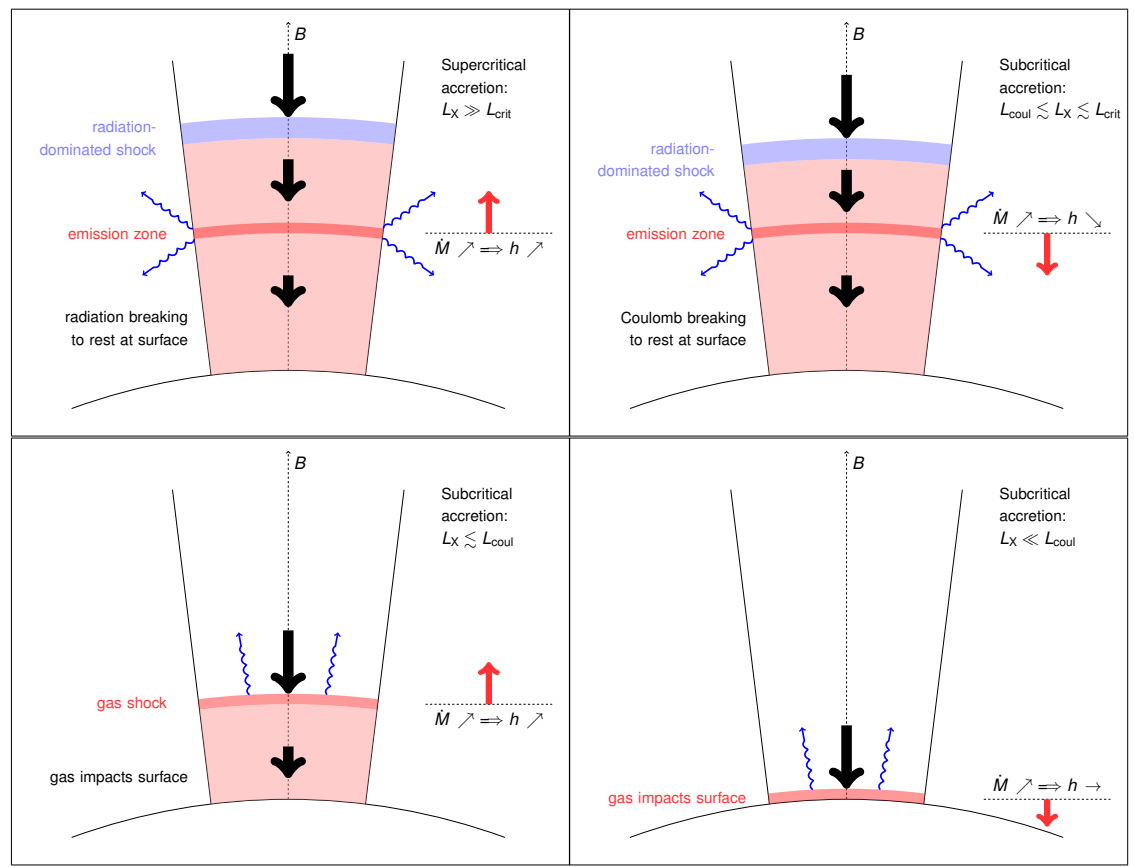

Figure 3. The different modes of accretion as a function of luminosity [after 59].

which is typically located around $2000 \mathrm{~km}$ from the neutron star. Magnetic torques couple the rotation of the neutron star and the rotation of the material at the Alfvén radius. This means that there should be a direct relation between the X-ray pulsational period and the parameters determining $r_{\text {mag }}$ [34-37]. Dramatic spin-up episodes are seen, for example, during giant outbursts of Be X-ray binaries [3, 3840], while many sources show extended spin up/spin down phases indicating that they are close to equilibrium $[18,41,42]$. These observations allow, in principle, an independent check of magnetic fields obtained from cyclotron lines (see Sect. 3 and [43]).

On shorter timescales, for high accretion rates, giant flares where sources suddenly brighten by a factor of 10 and more for a few pulsation periods (10s to 100s of seconds), and off states, where the X-ray flux suddenly shuts down for a short while, are seen in many luminous HMXB [44-46]. These are interpreted as evidence that accretion is inhibited by the propeller effect, where the accreted material cannot overcome the centrifugal barrier imposed by the rotating magnetic field $[11,47$, and references therein]. They are probably related to the flaring seen in supergiant fast X-ray transients.

\section{The Accretion Column}

\subsection{Theoretical Picture}

From the Alfvén radius onwards the matter follows the $B$-field lines and falls with a free fall speed of $\sim 0.7 c$ onto the magnetic poles of the neutron star where it is decelerated to $v=0 \mathrm{~cm} \mathrm{~s}^{-1}$ and therefore has to radiate its kinetic energy away. Since the material is confined to the magnetic field, the foot point of this accretion column has a radius of $r_{0} \sim 1 \mathrm{~km}$. 
How the accreted material is stopped depends strongly on the luminosity emitted at the polar caps, i.e., on $\dot{M}$. Building on earlier work [e.g., 48-58, and references therein], Becker et al. [59] have recently developed a scenario describing the different regimes of accretion in the accretion column. At the highest luminosities radiation pressure dominates (Fig. 3). This is the case for

$$
L_{\mathrm{X}} \gtrsim L_{\text {crit }}=2.72 \times 10^{38} \operatorname{erg} \mathrm{s} \frac{\sigma_{\mathrm{T}}}{\sqrt{\sigma_{\perp} \sigma_{\|}}}\left(\frac{M}{1 M_{\odot}}\right) \frac{r_{0}}{R}
$$

where $\sigma_{\mathrm{T}}$ is the Thomson cross section and $\sigma_{\perp}$ and $\sigma_{\|}$are the cross sections for scattering perpendicular and parallel to the $B$-field. As $L_{\mathrm{X}}$ decreases, Coulomb interaction becomes dominant, while at even lower $L_{\mathrm{X}}$, gas pressure and eventually scattering in the surface of the neutron star are the most important processes to stop the material. The resulting continuum is a combination of soft bremsstrahlung photons, as well as harder photons from bulk motion Comptonization in the decelerating accretion flow. Of lesser importance are black body radiation from the surface of the neutron star and cyclotron emission [49]. The resulting continuum shape is roughly a power law with an exponential cutoff.

The most important diagnostics to test these ideas are observations of cyclotron lines. While the electron energy distribution parallel to the $B$-field lines is essentially thermal, the motion of electrons perpendicular to the $\sim 10^{12} \mathrm{G} B$-field at the polar caps is quantized into Landau levels. The energy of these Landau levels is [e.g., 61]

$$
E_{n}=m_{\mathrm{e}} c^{2} \frac{\sqrt{1+2 n\left(B / B_{\text {crit }}\right) \sin ^{2} \theta}-1}{\sin ^{2} \theta} \quad \text { where } \quad B_{\text {crit }}=\frac{m_{\mathrm{e}}^{2} c^{3}}{e \hbar} \sim 4.4 \times 10^{13} \mathrm{G}
$$

$B_{\text {crit }}$ is the quantum-electrodynamical critical magnetic field. As a result of the quantization, the cross section for the scattering of photons off these electrons is strongly increased at the resonance energies corresponding to the energy differences between the Landau levels. For $B \ll B_{\text {crit }}$ the Landau levels are approximately equidistant with an energy difference of

$$
E_{\mathrm{cyc}}=\frac{\hbar e}{m_{\mathrm{e}} c} B=11.6 \mathrm{keV}\left(\frac{B}{10^{12} \mathrm{G}}\right)
$$

Figure 4 shows the cross section for the scattering of photons off these quantized electrons [60, 6264]. Note the extremely large dynamic range of the cross section as well as the very strong dependence on the scattering angle, which makes the numerical treatment of radiation transport in accretion columns very difficult. Both, Monte Carlo simulations [62, 63, 65-68] as well as radiative transfer calculations $[55-58,69,70]$ show that as a result of resonance scattering complex absorption line like features, "cyclotron line scattering features" or "cyclotron lines" are formed for a variety of physical circumstances. See the contributions by Schwarm and Schönherr in these proceedings for further information on the theory of cyclotron line formation. Cyclotron lines are of major observational importance since the determination of their energy allows us to measure directly the $B$-field strength at the location where they were formed. Not only is this the only direct way to measure neutron star magnetic fields, but in addition observations of cyclotron lines also allow us to test the models for the accretion flow onto neutron stars discussed above.

\subsection{Observations of Cyclotron Line Behavior}

Because of the difficulties in obtaining a fully self consistent model for the radiation emerging from the accretion column, the spectra of accreting neutron stars are often modeled with empirical models 

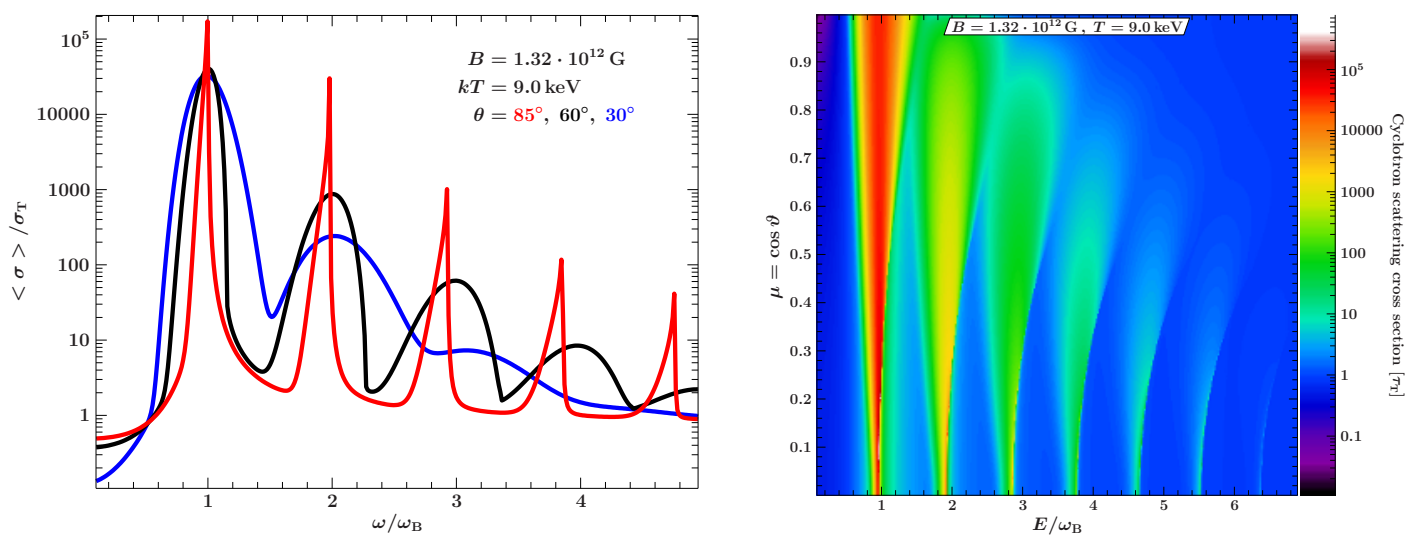

Figure 4. Cross section for resonant Compton scattering for a magnetic field of $B=0.03 B_{\text {crit }}=1.32 \times 10^{12} \mathrm{G}$ and an electron temperature of $k T_{\mathrm{e}}=9 \mathrm{keV}$ [60]. Left: Cross section for incident photon angles of $30^{\circ}, 60^{\circ}$, and $85^{\circ}$ with respect to the magnetic field. Right: Color coded cross section showing the strong dependence of the cross section on the scattering angle.

which try to approximate the expected continuum shape with simpler formulations ${ }^{2}$. Müller et al. [72] give a comprehensive overview of these spectral models. In addition to the simple exponentially cutoff power law model,

$$
\operatorname{CUTOFFPL}(E) \propto E^{-\Gamma} \exp \left(-E / E_{\text {fold }}\right)
$$

the two most commonly used continua are the Negative Positive Exponential Powerlaw, NPEX [73], and the Fermi Dirac Cutoff [75]:

$$
\operatorname{NPEX}(E) \propto\left(E^{-\Gamma_{1}}+a E^{+\Gamma_{2}}\right) \exp \left(-E / E_{\text {fold }}\right) \quad \text { and } \operatorname{FDCUT}(E) \propto \frac{E^{-\Gamma}}{1+\exp \left(\left(E-E_{\text {break }}\right) / E_{\text {fold }}\right)}
$$

where $\Gamma_{i}$ are the photon indices (in NPEX, one often fixes $\Gamma_{2}=2$; [e.g., 74]), $E_{\text {break }}$ is called the "break energy" and $E_{\text {fold }}$ is called the "folding energy". Note that the term "cutoff energy" is sometimes used for either the folding or the break energy, which can lead to confusion.

When data modeling the basic continuum model is modified by absorption in the stellar wind and the interstellar medium. If fluorescence emission is important it is described by additional Gaussian emission components. Even though cyclotron lines are predicted to have complex shape [e.g., 65, and references therein], due to the moderate resolution of most hard X-ray detectors the deviations from a simple line profile are often not resolved and it is often sufficient to describe the cyclotron line with multiplicative Gaussian or Lorentzian absorption profiles of the form

$$
F(E) \propto \operatorname{CONT}(E) \cdot \exp (-\tau(E)) \quad \text { with } \quad \tau(E)=\tau_{\mathrm{CRSF}} \exp \left(-\frac{1}{2}\left(\frac{E-E_{\mathrm{CRSF}}}{\sigma_{\mathrm{CRSF}}}\right)^{2}\right)
$$

where $\operatorname{CONT}(E)$ is one of the continuum models discussed above.

Starting with the initial discovery of a cyclotron line in the X-ray spectrum of Her X-1 [76], cyclotron lines have so far been detected in the spectra of $\sim 20$ neutron stars. Caballero and Wilms

\footnotetext{
${ }^{2}$ The only application of a self consistent model is the work of Ferrigno et al. [71] on 4U0115+63. The main problem of such models is the large number of free parameters combined with numerical instabilities during $\chi^{2}$ fitting. A more stable version of such a continuum modeling code is expected to be available in 2014 (Wolff et al., priv. comm.).
} 


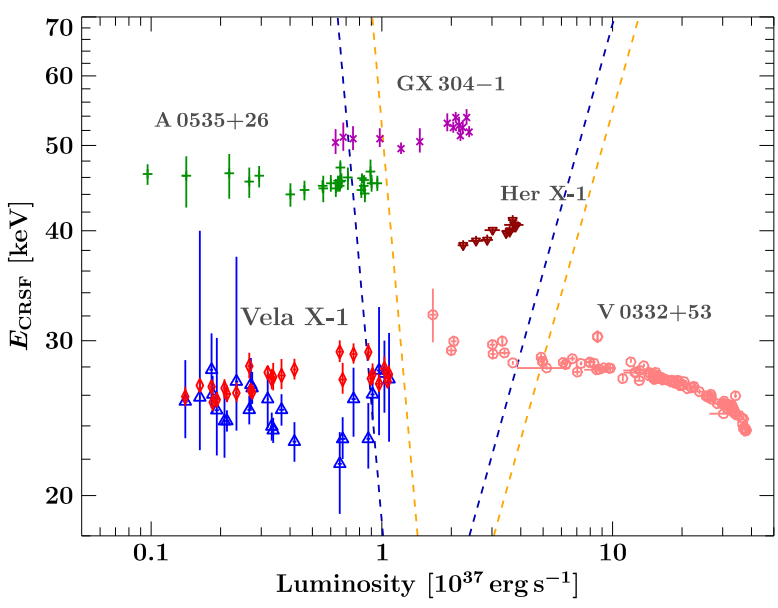

Figure 5. Luminosity dependent location of cyclotron lines [after 85]. The dashed lines separate the radiation dominated, Coulomb dominated, and gas pressure dominated regions for a neutron star mass of $1.4 M_{\odot}$ (blue) and $1.8 M_{\odot}$ (orange; this is the mass of the neutron star in Vela X-1). For Vela X-1, the blue data points show the energy of the fundamental line, while the red data points are the energy of the first harmonic line divided by two.

[77] and Pottschmidt et al. [78] give an overview of the observational status, including tables with relevant information on the systems. The discovery rate of new cyclotron line sources is 1-2 per year, and dominated by the rate with which new Be sources are going into outburst. The variety of accretion environments in which the sources are located means that the statistics of directly measured neutron star magnetic fields is still dominated by small number statistics. Continued target of opportunity observations of new and already known transients are of crucial importance to make further progress. Perhaps the most important results of the existing observations are the following:

Cyclotron lines are phase dependent: As the neutron star rotates around its axis, our line of sight onto the column changes. Pulse phase dependent observations of the spectrum therefore allow us to probe effects caused by the strong dependence of the scattering cross section on the scattering angle (Fig. 4). The observed phase dependence of the cyclotron line parameters (and the continuum) is complex, probably due to the fact that due to gravitational light bending we are observing a mixture of the emission from both magnetic poles. Examples of this behavior are Her X-1 [79, 80] and GX 301-2 [81]. A promising new technique to study this behavior based on the phase lag of the line has recently been suggested by Ferrigno et al. [82]. See Schönherr et al. (these proceedings) for details.

Cyclotron lines are (too?) smooth: With the availability of NUSTAR [83] low background spectra with good energy resolution are now becoming available. With $0.9 \mathrm{keV}$ at $60 \mathrm{keV}$ these are good enough to reveal structure in the cyclotron lines, such as the emission wings in the fundamental line predicted by CRSF models. Earlier observations with RXTE had revealed indications for complex lines [e.g., in V0332+53, 84], but such observations were only possible for the broadest, deepest lines. For Her X-1 and Vela X-1 the NUSTAR data suggest much less structure than what has been predicted $[80,85]$. A possible explanation is that the line is formed in a thicker layer in the accretion column than expected, and the $B$-field gradient or velocity gradients will smooth out the features.

Cyclotron line energies are luminosity dependent: Because the cyclotron line energy is representative of the magnetic field strength at the location where the line is formed, we expect its energy to change with changes in the structure of the accretion column. After initial searches for secular changes in the line had already been successful [e.g., 74, 86, 87], the most convincing indication for a strong luminosity dependence of the cyclotron line were INTEGRAL observations of the 2004/2005 outburst of V0332+35, where Tsygankov et al. $[88,89]$ observed a strong decrease in the cyclotron line energy as the source increased in luminosity [see also 90, 91]. No hysteresis was found, i.e., the line energy was only dependent on the luminosity (Fig. 5). The explanation of this behavior is that during the brightest stages of the outburst the source was close to $L_{\text {crit }}$ (eq. 4 ). Here, as the lumi- 
nosity increases the radiative shock where the line is formed moves away from the neutron star due to increased radiation pressure. Since the $B$-field decreases with height, we then expect the line to move to lower energies (Fig. 3 and [59, 74, 86, 92]). Sources at lower luminosity such as Her X-1 or GX 304-1 show the opposite behavior [e.g., 93, 94], again as expected since these sources are in the regime where Coulomb interactions dominate and the column reduces its height as $\dot{M}$ rate increases.

A very interesting example is 4U0115+63. Earlier work had shown the line to decrease with flux [74] and strong hysteresis effects had been suggested [95]. As shown by Müller et al. [72, see also [96]], this behavior is due to continuum variation: in combination with broad cyclotron lines the NPEX continuum model can describe the broad band spectral shape well, however, the cyclotron line component only partly describes the real cyclotron lines but rather "deforms" the spectral continuum to obtain the proper continuum shape. Other continuum models, however, recover the correct behavior of the cyclotron line, which in this source is then found to be independent of luminosity. It turns out that this result is rather good for column models: The $B$-field of $4 \mathrm{U} 0115+63$ is so small that one would not expect this source to enter the radiation dominated regime, and therefore it would be difficult to explain the previously claimed behavior. At the same time, however, the case of 4U0115+63 illustrates again the need for a more physics based continuum model for accreting, magnetized neutron stars.

In summary, accreting neutron stars are a very exciting and promising laboratory for studying the behavior of matter under very exotic conditions. The diagnostic capabilities of the instrumentation on XMM-Newton, INTEGRAL, Chandra, and Suzaku has been recently complemented by the broad-band capabilities of NUSTAR, which will significantly add more information on the behavior of cyclotron lines. With the soon to be launched ASTROSAT mission, as well as with missions currently under consideration such as LOFT and ATHENA+, significant further progress is to be expected on these fascinating sources.

\section{Acknowledgements}

I thank the organizers for giving me the opportunity to participate in the workshop. It is a pleasure to thank all members of the MAGNET collaboration for the input and discussions in recent years. This work has been partially funded by Deutsches Zentrum für Luft- und Raumfahrt under grant numbers 50 OR 1113 and 50 OR 1207.

\section{References}

[1] N.I. Shakura, R. Sunyaev, A\&A 24, 337 (1973)

[2] R. Staubert, D. Klochkov, J. Wilms, A\&A 500, 883 (2009)

[3] C.A. Wilson, M.H. Finger, A. Camero-Arranz, ApJ 678, 1263 (2008)

[4] D.M. Popper, ARA\&A 18, 115 (1980)

[5] H. Bondi, F. Hoyle, MNRAS 104, 273 (1944)

[6] J.M. Blondin, J.W. Woo, ApJ 445, 889 (1995)

[7] A. Manousakis, R. Walter, J.M. Blondin, A\&A 547, A20 (2012)

[8] J.E. Pringle, A.R. King, Astrophysical Flows (Cambridge Univ. Press, Cambridge, 2007)

[9] H.J.G.L.M. Lamers, J.P. Cassinelli, Introduction to Stellar Winds (Cambridge Univ. Press, Cambridge, 1999)

[10] J. Frank, A. King, D. Raine, Accretion Power in Astrophysics, 2nd edn. (Cambridge Univ. Press, Cambridge, 1992)

[11] E. Bozzo, M. Falanga, L. Stella, ApJ 683, 1031 (2008)

[12] P. Reig, Ap\&SS 332, 1 (2011) 
[13] R.W. Hanuschik, A\&A 308, 170 (1996)

[14] A. Slettebak, PASP 100, 770 (1988)

[15] M. Kühnel, S. Müller, I. Kreykenbohm, F. Fürst et al., A\&A 555, A95 (2013)

[16] W.A. Heindl, W. Coburn, D.E. Gruber, R.E. Rothschild et al., ApJ 563, L35 (2001)

[17] S. Müller, M. Kühnel, I. Caballero, K. Pottschmidt et al., A\&A 546, A125 (2012)

[18] A. González-Galán, E. Kuulkers, P. Kretschmar, S. Larsson et al., A\&A 537, A66 (2012)

[19] R.H.D. Corbet, J.L. Sokoloski, K. Mukai, C.B. Markwardt et al., ApJ 675, 1424 (2008)

[20] C. Ferrigno, A. Segreto, A. Santangelo, J. Wilms et al., A\&A 462, 995 (2007)

[21] D. Chakrabarty, M.H. van Kerkwijk, J.E. Larkin, ApJ 497, L39 (1998)

[22] D.M. Marcu, F. Fürst, K. Pottschmidt, V. Grinberg et al., ApJ 742, L11 (2011)

[23] F. Fürst, D.M. Marcu, K. Pottschmidt, et al., Proc. of Science INTEGRAL 2010, 017 (2011)

[24] N. Shakura, K. Postnov, A. Kochetkova, L. Hjalmarsdotter, MNRAS 420, 216 (2012)

[25] K. Postnov, Proc. of Science INTEGRAL 2010, 015 (2011)

[26] F. Fürst, I. Kreykenbohm, K. Pottschmidt, J. Wilms et al., A\&A 519, 37 (2010)

[27] S. Suchy, K. Pottschmidt, J. Wilms, I. Kreykenbohm et al., ApJ 675, 1487 (2008)

[28] F. Fürst, S. Suchy, I. Kreykenbohm, L. Barragán et al., A\&A 535, A9 (2011)

[29] S. Watanabe, N. Nagase, M. Sako, M. Ishida et al., Adv. Space Res. (2006), in press

[30] N.S. Schulz, C.R. Canizares, J.C. Lee, M. Sako, ApJ 564, L21 (2002)

[31] F. Nagase, G. Zylstra, T. Sonobe, T. Kotani et al., ApJ 436, L1 (1994)

[32] F. Nagase, S. Hayakawa, N. Sato, K. Masai et al., PASJ 38, 547 (1986)

[33] K. Davidson, J.P. Ostriker, ApJ 179, 585 (1973)

[34] P. Ghosh, F.K. Lamb, ApJ 223, L83 (1978)

[35] P. Ghosh, F.K. Lamb, ApJ 232, 239 (1979)

[36] R.W. Nelson, L. Bildsten, D. Chakrabarty, M.H. Finger et al., ApJ (1998), in press

[37] R. Perna, E. Bozzo, L. Stella, ApJ 639, 363 (2006)

[38] L. Bildsten, D. Chakrabarty, J. Chiu, M.H. Finger et al., ApJS 113, 367 (1997)

[39] D.T. Koh, L. Bildsten, D. Chakrabarty, R.W. Nelson et al., ApJ 479, 933 (1997)

[40] I. Caballero, K. Pottschmidt, D. Marcu, L. Barragan et al., ApJ 764, L23 (2013)

[41] S. Fritz, I. Kreykenbohm, J. Wilms, R. Staubert et al., A\&A 458, 885 (2006)

[42] S.Ç. Inam, Ş. Şahiner, A. Baykal, MNRAS 395, 1015 (2009)

[43] N.R. Ikhsanov, M.H. Finger, ApJ 753, 1 (2012)

[44] I. Kreykenbohm, J. Wilms, P. Kretschmar, J.M. Torrejón et al., A\&A 492, 511 (2008)

[45] P. Reig, T. Belloni, G.L. Israel, S. Campana et al., A\&A 485, 797 (2008)

[46] E. Göğüş, I. Kreykenbohm, T.M. Belloni, A\&A 525, L6 (2011)

[47] W. Cui, ApJ 482, L163 (1997)

[48] P.A. Becker, M.T. Wolff, ApJ 621, L45 (2005)

[49] P.A. Becker, M.T. Wolff, ApJ 654, 435 (2007)

[50] S.G. Alexander, J. Davila, D.J. Dimattio, ApJ 459, 666 (1996)

[51] D.J. Burnard, R.I. Klein, J. Arons, ApJ 349, 262 (1990)

[52] D.J. Burnard, J. Arons, R.I. Klein, ApJ 367, 575 (1991)

[53] S.J. Sturner, C.D. Dermer, A\&A 284, 161 (1994)

[54] J. Arons, R.I. Klein, S.M. Lea, ApJ 312, 666 (1987)

[55] P. Mészáros, W. Nagel, ApJ 298, 147 (1985)

[56] P. Mészáros, W. Nagel, ApJ 299, 138 (1985) 
[57] W. Nagel, ApJ 251, 278 (1981)

[58] W. Nagel, ApJ 251, 288 (1981)

[59] P.A. Becker, D. Klochkov, G. Schönherr, O. Nishimura et al., A\&A 544, A123 (2012)

[60] F.W. Schwarm, G. Schönherr, J. Wilms et al., A\&A (2013), to be submitted

[61] P. Mészáros, High-energy radiation from magnetized neutron stars (Chicago Univ. Press, Chicago, 1992)

[62] R.A. Araya, A.K. Harding, ApJ 517, 334 (1999)

[63] R.A. Araya-Góchez, A.K. Harding, ApJ 544, 1067 (2000)

[64] R. Sina, Ph.D. thesis, Univ. Maryland, College Park (1996)

[65] G. Schönherr, J. Wilms, P. Kretschmar, I. Kreykenbohm et al., A\&A 472, 353 (2007)

[66] M. Isenberg, D.Q. Lamb, J.C.L. Wang, ApJ 493, 154 (1998)

[67] M. Isenberg, D.Q. Lamb, J.C.L. Wang, ApJ 505, 688 (1998)

[68] J.C.L. Wang, D.Q. Lamb, T.J. Loredo, I.M. Wasserman et al., Phys. Rev. Lett. 63, 1550 (1989)

[69] O. Nishimura, ApJ 672, 1127 (2008)

[70] O. Nishimura, PASJ 57, 769 (2005)

[71] C. Ferrigno, P.A. Becker, A. Segreto, T. Mineo et al., A\&A 498, 825 (2009)

[72] S. Müller, C. Ferrigno, M. Kühnel, G. Schönherr et al., A\&A 551, A6 (2013)

[73] K. Makishima, T. Mihara, F. Nagase, Y. Tanaka, ApJ 525, 978 (1999)

[74] M. Nakajima, T. Mihara, K. Makishima, H. Niko, ApJ 646, 1125 (2006)

[75] Y. Tanaka, in Hydrodynamics of Stars and Compact Objects, edited by D. Mihalas, K.H.A. Winkler (Springer, 1986), p. 198

[76] J. Trümper, W. Pietsch, C. Reppin, W. Voges et al., ApJ 219, L105 (1978)

[77] I. Caballero, J. Wilms, Mem. Soc. Astron. Ital. 83, 230 (2012)

[78] K. Pottschmidt, S. Suchy, E. Rivers, R.E. Rothschild et al., in SUZAKU 2011: Exploring the $X$-ray Universe: Suzaku and Beyond, edited by R. Petre, K. Mitsuda, L. Angelini (2012), AIP Conf. Ser. 1427, pp. 60-67

[79] D. Vasco, R. Staubert, D. Klochkov, A. Santangelo et al., A\&A 550, A111 (2013)

[80] F. Fürst, B.W. Grefenstette, R. Staubert et al., ApJ (2013), in press (arXiv:1309.5361)

[81] S. Suchy, F. Fürst, K. Pottschmidt, I. Caballero et al., ApJ 745, 124 (2012)

[82] C. Ferrigno, M. Falanga, E. Bozzo, P.A. Becker et al., A\&A 532, A76 (2011)

[83] F.A. Harrison, W.W. Craig, F.E. Christensen, C.J. Hailey et al., ApJ 770, 103 (2013)

[84] K. Pottschmidt, I. Kreykenbohm, J. Wilms, W. Coburn et al., ApJ 634, L97 (2005)

[85] F. Fürst, K. Pottschmidt, J. Wilms, J.A. Tomsick et al., ApJ (2013), in press (arXiv:1311.5514)

[86] T. Mihara, K. Makishima, F. Nagase, Adv. Space Res. 22, 987 (1998)

[87] D.E. Gruber, W.A. Heindl, R.E. Rothschild, W. Coburn et al., ApJ 562, 499 (2001)

[88] S.S. Tsygankov, A.A. Lutovinov, E.M. Churazov, R.A. Sunyaev, MNRAS 371, 19 (2006)

[89] S.S. Tsygankov, A.A. Lutovinov, A.V. Serber, MNRAS 401, 1628 (2010)

[90] I. Kreykenbohm, N. Mowlavi, N. Produit, S. Soldi et al., A\&A 433, L45 (2005)

[91] M. Nakajima, T. Mihara, K. Makishima, ApJ 710, 1755 (2010)

[92] T. Mihara, Y. Terada, M. Nakajima et al., Prog. Theor. Phys. Suppl. 169, 191 (2007)

[93] R. Staubert, N.I. Shakura, K. Postnov, J. Wilms et al., A\&A 465, L25 (2007)

[94] D. Klochkov, V. Doroshenko, A. Santangelo, R. Staubert et al., A\&A 542, L28 (2012)

[95] S. Müller, M. Obst, I. Kreykenbohm et al., Proc. of Science INTEGRAL 2010, 116 (2011)

[96] P.A. Boldin, S.S. Tsygankov, A.A. Lutovinov, Ast. Lett. 39, 375 (2013) 